\title{
Commentary: Another Dutch treat
}

\author{
Marco A. Zenati, MD, ${ }^{\mathrm{a}, \mathrm{b}}$ and Steven J. Yule, $\mathrm{PhD}^{\mathrm{b}, \mathrm{c}}$
}

\footnotetext{
From the ${ }^{a}$ Division of Cardiac Surgery, Veterans Affairs Boston Healthcare System; ${ }^{\mathrm{b}}$ Department of Surgery, Brigham and Women's Hospital, Harvard Medical School; and ' $S T R A T U S$ Center for Medical Simulation, Brigham and Women's Hospital, Boston, Mass.

Disclosures: Authors have nothing to disclose with regard to commercial support.

Received for publication Aug 28, 2019; revisions received Aug 28, 2019; accepted for publication Aug 28, 2019; available ahead of print Oct 17, 2019.

Address for reprints: Marco A. Zenati, MD, Division of Cardiac Surgery, Veterans Affairs Boston Healthcare System, 1400 VFW Parkway, Boston, MA 02132 (E-mail: Marco_Zenati@hms.harvard.edu).

J Thorac Cardiovasc Surg 2020;159:1893-4

0022-5223/\$0.00

Published by Elsevier Inc. on behalf of The American Association for Thoracic Surgery

https://doi.org/10.1016/j.jtcvs.2019.08.123
}

The Dutch have adapted over centuries to live and thrive on a land that lies partially below sea level. Out of necessity, they have developed innovative hydraulic engineering solutions that have kept their population safe; their model has been the source of inspiration for other countries. Following this tradition, Dutch authors have made major contributions to surgical patient safety (eg, Surgical Patient Safety System [SURPASS]).

In this new work by Spanjersberg and colleagues, ${ }^{2} 7$ nonacademic cardiac surgery centers in the Netherlands joined forces to test the hypothesis that a cardiac surgery-specific pre-incision time-out (Isala Safety Check [ISR]), in addition to the initial World Health Organization-style safety checklists, may improve outcomes. The proposed ISR requires that the 4 teams (surgery, anesthesia, perfusion, and nursing), and importantly each team leader, be physically present in the room and participate in the ISR time-out. The ISR is short, focused, and importantly designed by the professionals who use it, which likely enhanced adoption as use was not mandated to avoid an "administrative check-box" culture.

The authors state that the purpose of the study was not to investigate the mechanisms of improvement or what was fundamentally different in the procedures with ISR that resulted in enhanced outcomes but to make some sound suggestions that relate to both process improvement and team dynamics or "nontechnical skills." With ISR, the use of a transesophageal echo is mandatory, irrespective of the type of procedure, which reinforces evidence-based best practice. Each body system is addressed in search for potential "hazards" to the safe conduct of the procedure (eg, poorly controlled diabetes may trigger more frequent checks of blood glucose; chronic kidney disease may call for practice of goal-directed perfusion, etc), which may increase vigilance and team situation awareness. By allowing all team members to contribute their perspective during the ISR, the resulting improved teamwork and communication may have been the "secret sauce" that led to the observed operating room.

\section{References}

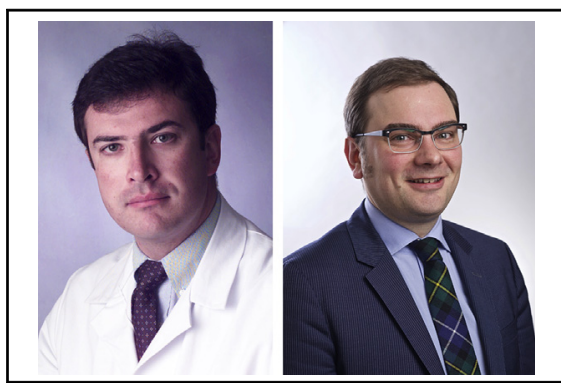

Marco A. Zenati, MD, and Steven J. Yule, PhD

Central Message

A cardiac surgery-specific pre-incision timeout is a reasonable addition to current strategies to improve teamwork and promote a climate of psychological safety and mutual trust in the cardiovascular operating room.

See Article page 1882 .

lower mortality. Trust among team members allows errors to be caught and recovered early, before an error cycle leads to patient harm.

Indeed, our research team has reported that indices of cognitive workload derived from heart rate variability among cardiac surgery team members tend to synchronize during pre-incision time-out, ${ }^{3}$ suggesting that the byproducts of an effective pre-incision time-out are (1) better shared mental model about the procedure about to start and proactive and (2) explicit strategies to avoid complications. This approach has been successfully applied to provide cognitive support to cardiac surgery teams during low-frequency/high-risk situations (eg, massive air embolism, protamine reaction, etc). ${ }^{4}$ The future of quality improvement is a multidisciplinary one, with data science, implementation science, and behavioral science working together to achieve improvements that clinical science cannot achieve alone. ${ }^{5}$ The proposed ISR has few drawbacks and only adds few minutes to the procedure. If these results are confirmed by other investigators, the addition of a systematic and procedure-specific pre-incision time-out seems destined to become a fixture in the cardiovascular

1. DeVries EN, Hollmann MW, Smorenburg SM, Gouma DJ, Boermeester MA Development and validation of the SURgical PAtient Safety System (SURPASS) checklist. Qual Saf Health Care. 2009;18:121-6.

2. Spanjersberg AJ, Ottervanger JP, Nierich AP, Speekenbrink RGH, Stooker W, Hoogendoorn M, et al. Implementation of a specific safety check is associated 
with lower postoperative mortality in cardiac surgery. J Thorac Cardiovasc Surg. 2020;159:1882-90.e2.

3. Kennedy-Metz LR, Dias RD, Leissner K, Yule SJ, Zenati MA. A formal preincision time-out promotes team workload synchronization in cardiac surgery. Presented at: Proceedings of the 16th Annual Society of Thoracic Surgeons' Multidisciplinary Cardiovascular and Thoracic Critical Care Conference, September 26-28, 2019; Baltimore, Md.
4. Tarola CL, Hirji S, Yule SJ, Gabany JM, Zenati A, Dias RD, et al. Cognitive support to promote shared mental models during safety-critical situations in cardiac surgery. 2018 IEEE Conf Cogn Comput Asp Situat Manag CogSIMA (2018). 2018;2018:165-7.

5. Zenati MA, Kennedy-Metz L, Dias RD. Cognitive engineering to improve patient safety and outcomes in cardiothoracic surgery. Semin Thor Cardiovasc Surg. 2020; 32(1):1-7. 